\title{
Super High-Selectivity Fifth-Order Bandpass Filter with Twelve Transmission Zeros
}

\author{
Yi-jun $C A I^{1}$, Feng-yu ZHANG ${ }^{2}$, Kai-da XU ${ }^{2,3,4}$, Dong-hao $L I^{2}$ \\ ${ }^{1}$ School of Optoelectronic and Communication Engineering, Xiamen University of Technology, Xiamen 361024, China \\ ${ }^{2}$ Dept. of Electronic Science, Xiamen University, Xiamen 361005, China \\ ${ }^{3}$ Shenzhen Research Institute of Xiamen University, Shenzhen 518057, China \\ ${ }^{4}$ Dept. of Electrical and Computer Engineering, University of Wisconsin-Madison, Madison, WI 53706, USA
}

kaidaxu@xmu.edu.cn

Submitted June 30, 2018 / Accepted October 5, 2018

\begin{abstract}
A fifth-order bandpass filter (BPF) with super high selectivity using three pairs of coupled lines and two open stubs is proposed. Twelve transmission zeros (TZs) from 0 to $2 f_{0}$ ( $f_{0}$ denotes center frequency of the passband) and five transmission poles (TPS) in the passband can be obtained to realize good out-of-band suppression and sharp roll-off skirts. For demonstration, a simple BPF prototype centered at $2.04 \mathrm{GHz}$ is designed, fabricated with measured 3-dB fractional bandwidth of $18 \%$ and very high transition band roll-off rates of over $567 \mathrm{~dB} / \mathrm{GHz}$. Good agreement between the simulations and measurements validates the design method.
\end{abstract}

\section{Keywords}

Bandpass filter, high-selectivity, coupled lines, transmission poles, transmission zeros

\section{Introduction}

High-selectivity bandpass filters (BPFs) with low insertion loss in the passband and high rejection in the stopband are drawing increasing attentions to meet the requirement of the modern wireless communication systems [1]. Recently, some works have been reported on introducing transmission zeros (TZs) to improve the out-of-band suppression of BPFs [2-13]. In [2-4], signal interference techniques have been invented for the generation of multiple TZs due to the construction of multiple transmission paths. In [5], a new source-load cross coupling type with both capacitive and inductive coupling is introduced to obtain multiple TZs for suppressing the undesired harmonics. Moreover, the concept of $\mathrm{TZ}$ resonator pair is proposed in [6], where four TZs are located at the resonant frequency points of the two resonator pairs. However, the lower stopband cannot reject very well because of its intrinsic limitation of the scheme. In [7], a BPF with four TZs using two open coupled lines and several stubs is proposed, where two TZs can be introduced with the help of the two shorted stubs to improve the frequency selectivity.
In this paper, a super high-selectivity fifth-order BPF using three pairs of coupled lines and two open stubs is proposed. Based on our previously reported work [8], two half-wavelength open stubs are loaded on the first and third pairs of coupled lines, respectively, to generate four more TZs at the out-of-band. Thus, the total number of TZs is increased to 12 at the frequency range from 0 to $2 f_{0}\left(f_{0}\right.$ is the required center frequency), which is the most number in the previously reported literature to the best of our knowledge. For demonstration, a filter example with center frequency of $2.04 \mathrm{GHz}$ is fabricated, whose simulated and measured results are in good agreement to validate the design idea.

\section{Design and Analysis of the Proposed BPF}

\subsection{Proposed BPF Design}

The ideal circuits of the proposed fifth-order BPF with twelve TZs, which consists of three pairs of coupled lines and two open stubs, is shown in Fig. 1. The middle (second) pair of $\lambda_{\mathrm{g}} / 4$ coupled lines (even/odd-mode charac-

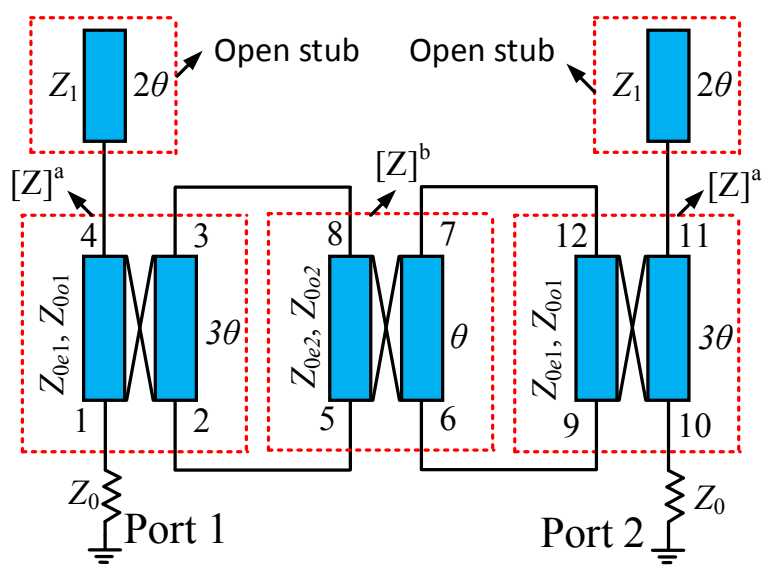

Fig. 1. Ideal circuit of the BPF with twelve TZs. 


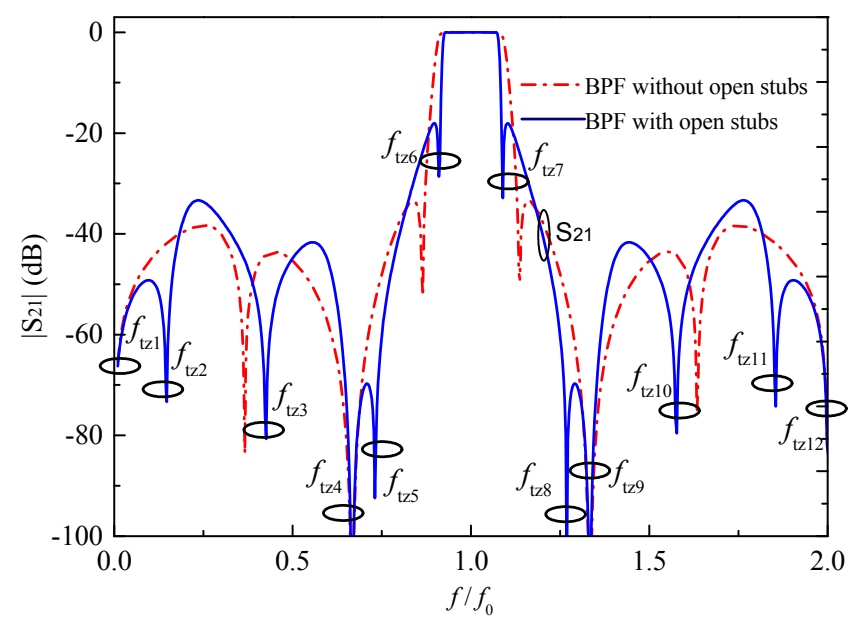

(a)

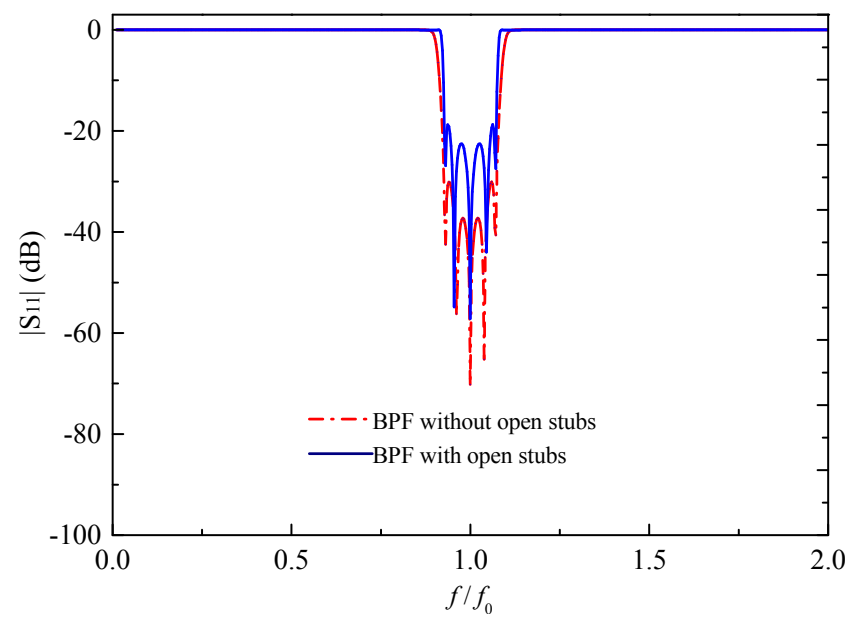

(b)

Fig. 2. Simulated results of the proposed ideal filter circuit: (a) $S_{21}$, (b) $S_{11}$, where $\theta=90^{\circ}, Z_{0}=50 \Omega, Z_{1}=90 \Omega$, $Z_{0 \mathrm{e} 1}=158 \Omega, Z_{001}=60 \Omega, Z_{0 \mathrm{e} 2}=109 \Omega, Z_{002}=65 \Omega$.

teristic impedance $\mathrm{Z}_{0 \mathrm{e} 2}, \mathrm{Z}_{0 \mathrm{o} 2}$, electrical length $\theta$ ) are connected to the first and third pairs of $3 \lambda_{\mathrm{g}} / 4$ coupled lines (even/odd-mode characteristic impedance $Z_{0 \mathrm{e} 1}, Z_{0 \mathrm{o}}$, electrical length $3 \theta$ ), and two $\lambda_{\mathrm{g}} / 2$ open stubs are loaded on the first and third pairs of coupled lines, respectively.

Figure 2 shows the simulated S-parameter comparisons between the proposed BPF (i.e., BPF with open stubs) and the BPF in [8] (i.e., BPF without open stubs). As seen in Fig. 2(a), four more TZs $\left(f_{\mathrm{tz} 2}, f_{\mathrm{tz} 5}, f_{\mathrm{tz} 8}, f_{\mathrm{tz} 11}\right)$ can be introduced by adding the two open stubs, and their positions are mainly determined by the characteristic impedance of the open stubs $Z_{1}$. For the previously generated eight TZs, the four TZs $f_{\mathrm{tz} 1}, f_{\mathrm{tz} 4}, f_{\mathrm{tz} 9}$ and $f_{\mathrm{tz} 12}$ keep unchanged whose positions can be expressed as

$$
f_{\mathrm{tz} 1}=0, \quad f_{\mathrm{tz} 4}=\frac{2}{3} f_{0}, \quad f_{\mathrm{tz} 9}=\frac{4}{3} f_{0}, \quad f_{\mathrm{tz} 12}=2 f_{0} .
$$

More importantly, the positions of the two TZs $f_{\mathrm{tz} 6}$ and $f_{\mathrm{tz} 7}$ are closer to the passband than the ones of BPF without open stubs. Thus, the roll-off skirt of the passband will become sharper to further improve frequency selectivity.

\subsection{Analysis of the Proposed BPF}

This filter circuit can also be analyzed by using impedance matrix deduction similarly to the method in [8]. From Fig. 1, we can obtain that $V_{2}=V_{5}, I_{2}=-I_{5}, V_{3}=V_{8}$, $I_{3}=-I_{8}, V_{6}=V_{9}, I_{6}=-I_{9}, V_{7}=V_{12}, I_{7}=-I_{12}$, and $I_{4}=I_{11}=$ $-\mathrm{j} \tan (2 \theta) \cdot V_{4} / Z_{1}$. $[\mathbf{Z}]^{\mathrm{a}}$ and $[\mathbf{Z}]^{\mathrm{b}}$ denote the impedance matrices of the $3 \lambda_{\mathrm{g}} / 4$ and $\lambda_{\mathrm{g}} / 4$ parallel-coupled lines, respectively. The overall impedance matrix $[\mathbf{Z}]^{\prime}$ and TZs of the filter can be calculated as

$$
\left[\begin{array}{c}
V_{1} \\
V_{10}
\end{array}\right]=\left[\begin{array}{ll}
Z_{11}^{\prime} & Z_{12}^{\prime} \\
Z_{21}^{\prime} & Z_{22}^{\prime}
\end{array}\right]\left[\begin{array}{c}
I_{1} \\
I_{10}
\end{array}\right] .
$$

Finally, twelve finite TZs in the stopband at the frequency range from 0 to $2 f_{0}$ can be obtained through calculation by setting transmission coefficient $S_{21}=0$, where $S_{21}$ is expressed as

$$
S_{21}=\frac{2 Z_{21}^{\prime} Z_{0}}{\left(Z_{11}^{\prime}+Z_{0}\right)\left(Z_{22}^{\prime}+Z_{0}\right)-Z_{12}^{\prime} Z_{21}^{\prime}} .
$$

For transmission poles (TPs), they can be calculated by setting reflection coefficient $S_{11}=0$, where $S_{11}$ can be expressed as

$$
S_{11}=\frac{\left(Z_{11}^{\prime}-Z_{0}\right)\left(Z_{22}^{\prime}+Z_{0}\right)-Z_{12}^{\prime} Z_{21}^{\prime}}{\left(Z_{11}^{\prime}+Z_{0}\right)\left(Z_{22}^{\prime}+Z_{0}\right)-Z_{12}^{\prime} Z_{21}^{\prime}} .
$$

Through calculation, five TPs can be obtained, as illustrated below

$$
\begin{gathered}
f_{\mathrm{tp} 1}=\frac{2 f_{0}}{\pi} \arccos \sqrt{\frac{Z_{0 \mathrm{e} 1}-2 Z_{0 \mathrm{o} 2}+Z_{0 \mathrm{o} 1}}{2 Z_{0 \mathrm{e} 1}+4 Z_{0 \mathrm{o} 2}+2 Z_{0 \mathrm{o} 1}}}, \\
f_{\mathrm{tp} 2}=\frac{2 f_{0}}{\pi} \arccos \sqrt{\frac{Z_{0 \mathrm{e} 1}-2 Z_{0 \mathrm{e} 2}+Z_{0 \mathrm{o} 1}}{2 Z_{0 \mathrm{e} 1}+4 Z_{0 \mathrm{e} 2}+2 Z_{0 \mathrm{o} 1}}}, \\
f_{\mathrm{tp} 3}=f_{0}, \\
f_{\mathrm{tp} 4}=\frac{2 f_{0}}{\pi}\left(\pi-\arccos \sqrt{\frac{Z_{0 \mathrm{e} 1}-2 Z_{0 \mathrm{e} 2}+Z_{0 \mathrm{o} 1}}{2 Z_{0 \mathrm{e} 1}+4 Z_{0 \mathrm{e} 2}+2 Z_{0 \mathrm{ol}}}}\right), \\
f_{\mathrm{tp} 5}=\frac{2 f_{0}}{\pi}\left(\pi-\arccos \sqrt{\frac{Z_{0 \mathrm{e} 1}-2 Z_{0 \mathrm{o} 2}+Z_{0 \mathrm{o} 1}}{2 Z_{0 \mathrm{e} 1}+4 Z_{0 \mathrm{o} 2}+2 Z_{0 \mathrm{o} 1}}}\right) .
\end{gathered}
$$

The positions of these five TPs are the same as those of the BPF without open stubs. This result can also be verified from the simulation comparison as shown in Fig. 2(b).

In addition to the TPs and TZs, the concerned BPF characteristics mainly include the $3-\mathrm{dB}$ fractional bandwidth $(\Delta f)$, maximal out-of-band $\left|S_{21}\right|\left(T_{\mathrm{s}}\right)$, maximal in-band $\left|\mathrm{S}_{11}\right|\left(T_{\mathrm{p}}\right)$ [4] and transition band roll-off rate $\left(\xi_{\mathrm{ROR}}\right)[14]$ referring to the responses in Fig. 3(a). Figure 3(b), (c) and (d) show the corresponding variations of $\Delta f, T_{\mathrm{s}}$ and $T_{\mathrm{p}}$ against the parameters $Z_{1}, k_{1}$ and $k_{2}$, respectively, where $k_{1}=\left(Z_{0 \mathrm{e} 1}-Z_{0 \mathrm{o} 1}\right) /\left(Z_{0 \mathrm{e} 1}+Z_{0 \mathrm{o} 1}\right), \quad k_{2}=\left(Z_{0 \mathrm{e} 2}-Z_{0 \mathrm{o} 2}\right) /\left(Z_{0 \mathrm{e} 2}+Z_{0 \mathrm{o} 2}\right)$. 
As plotted in Fig. 3(b), $T_{\mathrm{s}}$ and $T_{\mathrm{p}}$ will both decrease but $\Delta f$ will rise up when $Z_{1}$ increases. The maximum $\Delta f$ will be $18.7 \%$ when $Z_{1}$ increases to $180 \Omega$ under the return loss condition of over $10 \mathrm{~dB}$ within the passband. On the other hand, as $k_{1}$ increases in Fig. 3(c), $T_{\mathrm{s}}$ will grow up slightly but $T_{\mathrm{p}}$ will decrease and then rise up, while the bandwidth $\Delta f$ will fall down directly. In contrast, as $k_{2}$ increases, $T_{\mathrm{s}}$ will almost remain unchanged but $T_{\mathrm{p}}$ will reduce and then go up, while $\Delta f$ will rise up simultaneously as seen in Fig. 3(d).

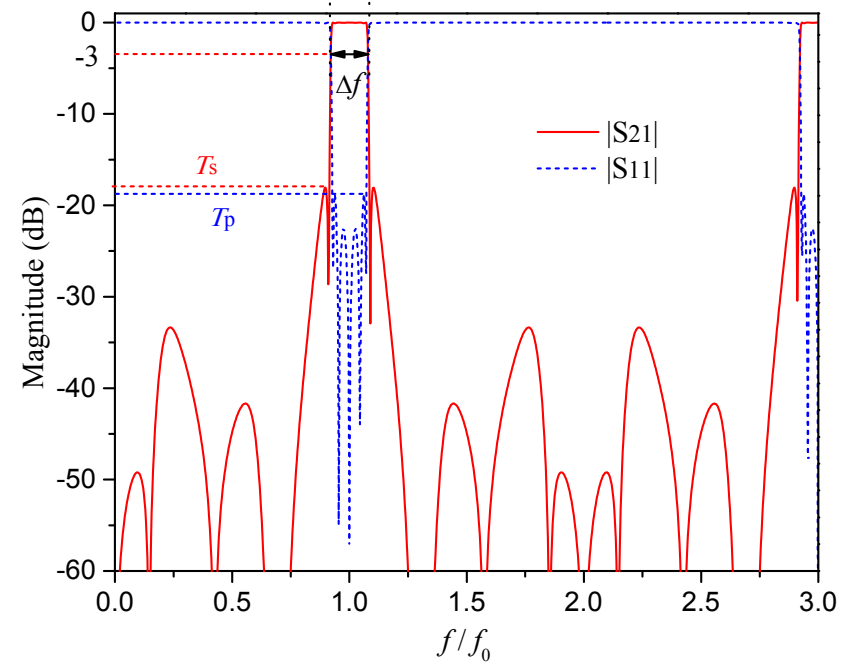

(a)

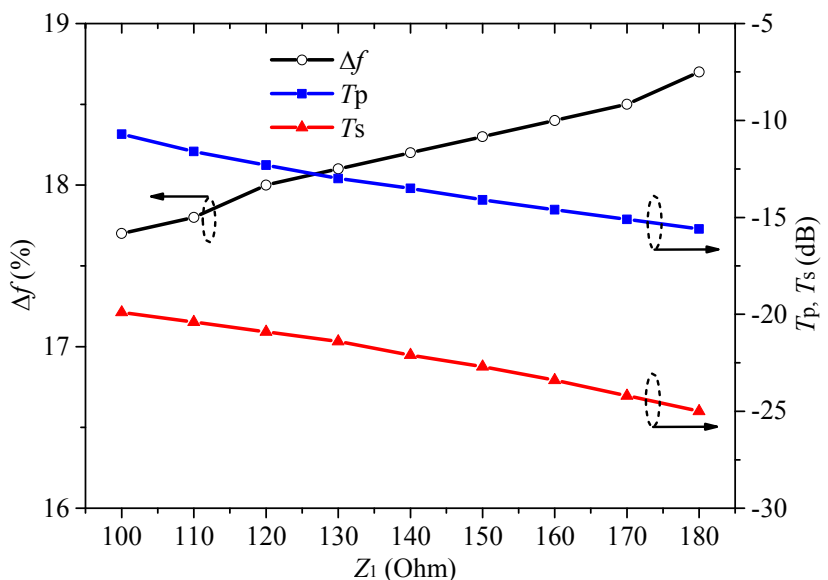

(b)

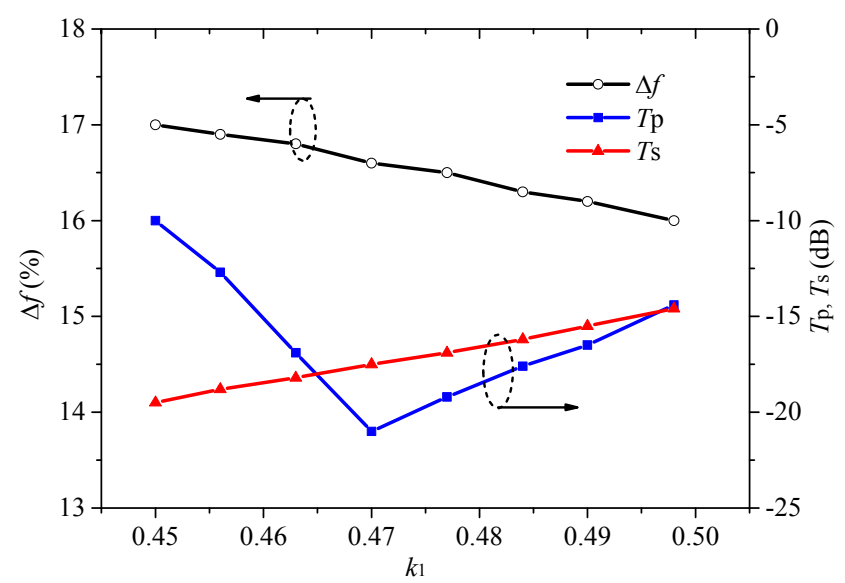

(c)

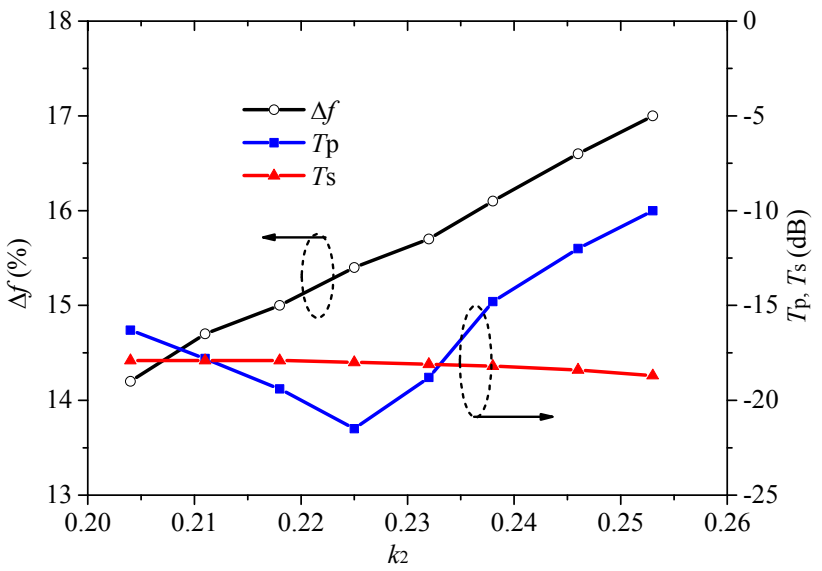

(d)

Fig. 3. Performance of the proposed BPF. (a) Definition of $T_{\mathrm{s}}$ and $T_{\mathrm{p}}$, and 3-dB bandwidth $\Delta f$. (b) $\Delta f, T_{\mathrm{s}}$ and $T_{\mathrm{p}}$ versus $Z_{1}\left(Z_{0 \mathrm{e} 1}=158 \Omega, Z_{001}=60 \Omega, Z_{0 \mathrm{e} 2}=109 \Omega\right.$, $\left.Z_{002}=65 \Omega\right)$, (c) $\Delta f, T_{\mathrm{s}}$ and $T_{\mathrm{p}}$ versus $k_{1}\left(Z_{1}=90 \Omega\right.$, $Z_{0 \mathrm{e} 2}=109 \Omega, Z_{002}=65 \Omega$ ), and (d) $\Delta f, T_{\mathrm{s}}$ and $T_{\mathrm{p}}$ versus $k_{2}\left(Z_{1}=90 \Omega, Z_{0 \mathrm{el}}=158 \Omega, Z_{0 \mathrm{ol}}=60 \Omega\right)$.

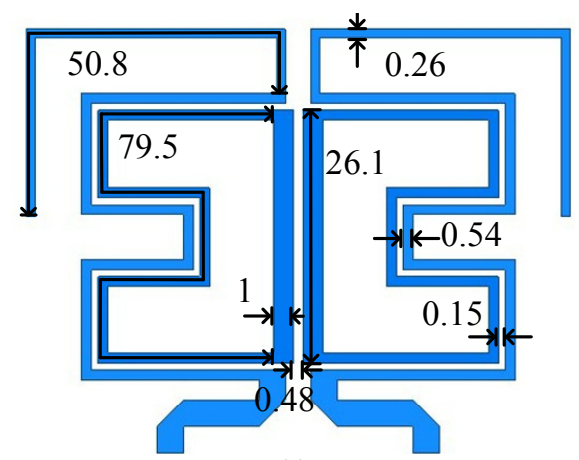

(a)

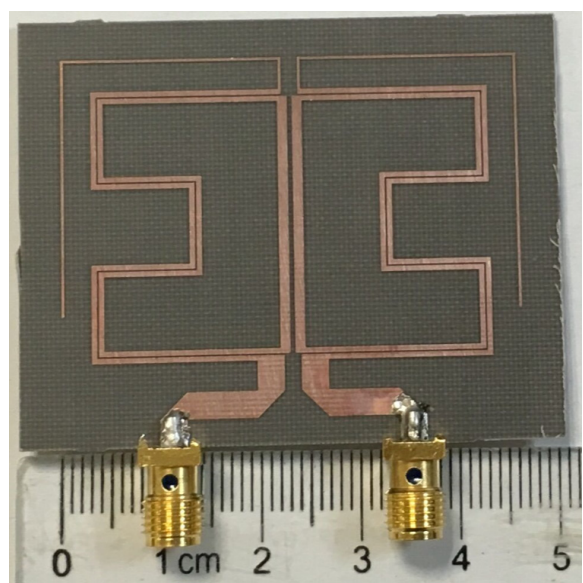

(b)

Fig. 4. (a) Layout of the proposed fifth-order BPF (unit: $\mathrm{mm}$ ) and (b) its fabricated photograph

\section{Implementation Results}

\subsection{Simulation and Measurement}

Based on the above analysis, the final parameters of the designed ideal circuit model filter with center frequency 
at $2.04 \mathrm{GHz}$ are taken as: $Z_{0}=50 \Omega, Z_{0 \mathrm{e} 1}=158 \Omega$, $Z_{0 \mathrm{o} 1}=60 \Omega, \quad Z_{0 \mathrm{e} 2}=109 \Omega, \quad Z_{0 \mathrm{o} 2}=68 \Omega, \quad Z_{1}=90 \Omega \quad$ and $\theta=90^{\circ}$. Figure 4 illustrates a verified physical layout of this wideband BPF as well as its photograph fabricated on the F4B substrate with relative permittivity of 2.65 and thickness of $1 \mathrm{~mm}$, where the loss tangent of dielectric is $\tan \delta=0.006$, the conductivity and thickness of copper conductor are $5.8 \times 10^{7} \mathrm{~S} / \mathrm{m}$ and $35 \mu \mathrm{m}$, respectively.

The measured S-parameters are plotted in Fig. 5 along with the simulation using Ansoft HFSS for comparisons. The simulated lower and upper transition band roll-off rates are better than $567 \mathrm{~dB} / \mathrm{GHz}$, while the measured counterparts are also over $567 \mathrm{~dB} / \mathrm{GHz}$. Table 1 tabulates the performance comparisons of the proposed BPF with some previous works, and it can be seen that the presented study has enhanced transition band roll-off skirts to realize super high frequency selectivity with the most number of TZs.

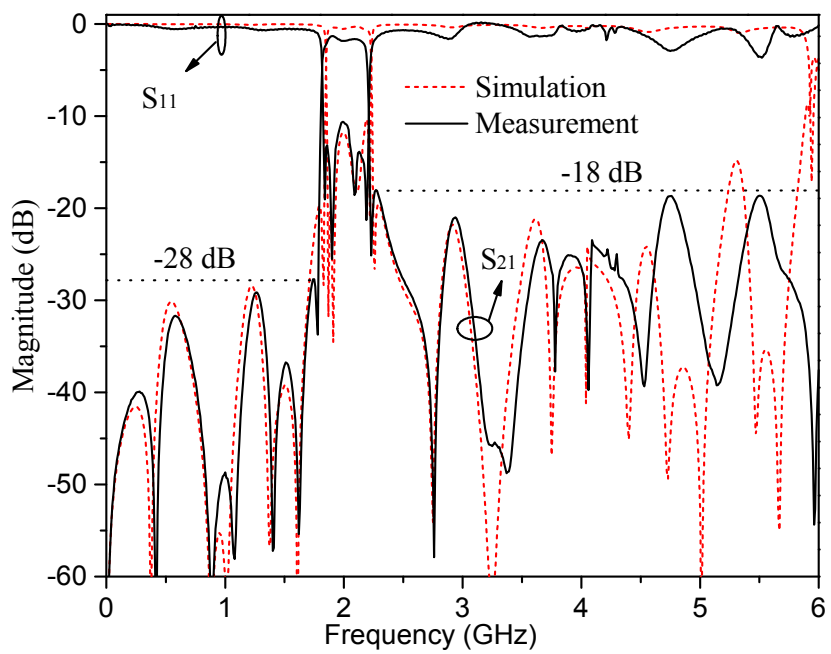

Fig. 5. Simulated and measured S-parameters of the BPF.

\begin{tabular}{|c|c|c|c|c|c|c|}
\hline Ref. & TPs & TZs & $\Delta \boldsymbol{f}$ & $\begin{array}{c}\xi_{\text {ROR }}{ }^{*} \\
(\boldsymbol{L} / \boldsymbol{U}) \\
(\mathbf{d B} / \mathbf{G H z})\end{array}$ & $\begin{array}{c}\text { Upper } \\
\text { stopband } \\
(\mathbf{d B})\end{array}$ & $\begin{array}{c}\text { Circuit } \\
\text { size } \\
\boldsymbol{\lambda}_{\mathrm{g}} \times \lambda_{\mathrm{g}}\end{array}$ \\
\hline$[4]-\mathrm{I}$ & 5 & 6 & $61.7 \%$ & $210 / 130$ & $>15\left(2.7 f_{0}\right)$ & $0.68 \times 0.45$ \\
\hline$[7]$ & 7 & 4 & $78 \%$ & $288 / 175$ & $>35.1\left(2.6 f_{0}\right)$ & $0.56 \times 0.23$ \\
\hline$[8]$ & 5 & 8 & $19 \%$ & $340 / 425$ & $>18\left(3 f_{0}\right)$ & $0.39 \times 0.28$ \\
\hline$[15]-\mathrm{A}$ & 5 & 6 & $70 \%$ & $81 / 121$ & $>21\left(2.6 f_{0}\right)$ & $0.53 \times 0.41$ \\
\hline$[15]-\mathrm{B}$ & 5 & 6 & $37 \%$ & $94 / 120$ & $>23\left(2.8 f_{0}\right)$ & $0.61 \times 0.55$ \\
\hline This work & $\mathbf{5}$ & $\mathbf{1 2}$ & $\mathbf{1 8 \%}$ & $\mathbf{5 6 7 / 5 6 7}$ & $>\mathbf{1 8}\left(\mathbf{3} \boldsymbol{f}_{\mathbf{0}}\right)$ & $\mathbf{0 . 4 5} \times \mathbf{0 . 3}$ \\
\hline
\end{tabular}

*Transition band roll-off rates $\boldsymbol{\xi}_{\mathrm{ROR}}=\left|\delta_{-20 \mathrm{~dB}}-\delta_{-3 \mathrm{~dB}}\right|\left|f_{-20 \mathrm{~dB}}-f_{-3 \mathrm{~dB}}\right|$, where $\delta_{-20 / 3 \mathrm{~dB}}$ denotes the $20 / 3 \mathrm{~dB}$ attenuation point, and $f_{-20 / 3 \mathrm{~dB}}$ is the $20 / 3 \mathrm{~dB}$ passband frequency of $\left|S_{21}\right| . L$ and $U$ denote lower and upper transition band roll-off rates, respectively

Tab. 1. Performance comparisons with some previous BPFs.

\subsection{Discussion}

For the ideal BPF circuit model in Fig. 1, the performance of the passbands and lower/upper stopbands must be ideal with symmetrical curves as seen in Fig. 2 by using the theoretically mathematical calculation or commercial soft- ware simulation (e.g., Ansoft Designer or ADS). For the physically realized geometry such as the layout in Fig. 4, however, the simulated or measured results will be deteriorated due to many discontinuities at the corners of two connected microstrip lines or meandering lines in the fullwave electromagnetic simulation, compared with the theoretically calculated results. Generally, at higher frequency, the discrepancy between full-wave simulated results and theoretically calculated results will be larger than that at lower frequency in the circuit design as seen in Fig. 5. Therefore, it is reasonable that the simulated and measured results of the physically realized geometry are different with the simulation of the ideal BPF circuit model. The upper stopband rejection level is worse than the lower stopband rejection level. The work in [8] also has the same situation as seen in Tab. 1.

\section{Conclusion}

This paper has presented a compact super high-selectivity fifth-order BPF with twelve TZs using simple circuit structure. The design idea and calculations on TZs and TPs are also provided. Due to its sharp roll-off skirts, multiple TPs and TZs, and ease of fabrication and integration with other circuits, the proposed BPF is promising in application of modern wireless communication systems.

\section{Acknowledgments}

This work was supported in part by the National Natural Science Foundation of China under Grant 61601390 and the Shenzhen Science and Technology Innovation Project under Grant JCYJ20170306141249935.

\section{References}

[1] HONG, J.-S., LANCASTER, M. J. Microstrip Filters for RF/Microwave Applications. New York: Wiley, 2001. ISBN: $0-471-38877-7$

[2] GOMEZ-GARCIA, R., ALONSO, J. I. Design of sharp-rejection and low-loss wide-band planar filters using signal-interference techniques. IEEE Microwave and Wireless Components Letters, 2005, vol. 15 , no. 8, p. 530-532. DOI: 10.1109/LMWC.2005.852797

[3] FENG, W. J., CHE, W. Q., XUE, Q. Transversal signal interaction: Overview of high-performance wideband bandpass filters. IEEE Microwave Magazine, 2014, vol. 15, no. 2, p. 84-96. DOI: 10.1109/MMM.2013.2296216

[4] FENG, W. J., CHE, W. Q., CHANG, Y. M., et al. High selectivity fifth-order wideband bandpass filters with multiple transmission zeros based on transversal signal-interaction concepts. IEEE Transactions on Microwave Theory and Techniques, 2013, vol. 61, no. 1, p. 89-97. DOI: 10.1109/TMTT.2012.2227785

[5] XU, K., ZHANG, Y., et al. Novel circular dual-mode filter with both capacitive and inductive source-load coupling for multiple transmission zeros. Journal of Electromagnetic Waves and Applications, 2012, vol. 26 no. 13, p. 1675-1684. DOI: 10.1080/09205071.2012.708966 
[6] XUE, Q., JIN, J. Y. Bandpass filters designed by transmission zero resonator pairs with proximity coupling. IEEE Transactions on Microwave Theory and Techniques, 2017, vol. 65, no. 11, p. 4103-4110. DOI: 10.1109/TMTT.2017.2697878

[7] XU, K. D., ZHANG, F. Y., LIU, Y. H., et al. High selectivity seventh-order wideband bandpass filter using coupled lines and open/shorted stubs. Electronic Letters, 2018, vol. 54, no. 4, p. 223-225. DOI: 10.1049/el.2017.4233

[8] XU, K. D., ZHANG, F. Y., LIU, Y. H., et al. Bandpass filter using three pairs of coupled lines with multiple transmission zeros. IEEE Microwave and Wireless Components Letters, 2018. vol. 28, no. 7, p. 576-578. DOI: 10.1109/LMWC.2018.2835643

[9] YANG, T., REBEIZ, G. M. Tunable 1.25-2.1-GHz 4-pole bandpass filter with intrinsic transmission zero tuning. IEEE Transactions on Microwave Theory and Techniques, 2015, vol. 63, no. 5, p. 1569-1578. DOI: 10.1109/TMTT.2015.2409061

[10] AI, J., ZHANG, Y., XU, K. D., et al. Miniaturized quint-band bandpass filter based on multi-mode resonator and $\lambda / 4$ resonators with mixed electric and magnetic coupling. IEEE Microwave and Wireless Components Letters, 2016, vol. 26, no. 5, p. 343-345. DOI: 10.1109/LMWC.2016.2549643

[11] XU, K. D., LI, M., LIU, Y. H., et al. Compact microstrip triplemode bandpass filters using dual-stub-loaded spiral resonators. Radioengineering, 2017, vol. 26, no. 1, p. 23-29. DOI: $10.13164 /$ re.2017.0023

[12] XU, K. D., BAI, Y., REN, X., XUE, Q. Broadband filtering power dividers using simple three-line coupled structures. IEEE Transactions on Components, Packaging and Manufacturing Technology, 2018, Early Access, p. 1-8. DOI: 10.1109/TCPMT.2018.2869077

[13] XU, K., LUO, Z., LIU, Y., LIU, Q. H. High-selectivity singleended and balanced bandpass filters using dual-mode ring resonators loaded with multiple stubs. AEÜ - International Journal of Electronics and Communications, 2018, vol. 96, p. 193-198. DOI: 10.1016/J.AEUE.2018.09.021

[14] AI, J., ZHANG, Y., XU, K. D., et al. Miniaturized frequency controllable bandstop filter using coupled line stubs loaded shorted SIR for tri-band application. IEEE Microwave and Wireless Components Letters, 2017, vol. 27, no. 7, p. 627-629. DOI: 10.1109/LMWC.2017.2711532

[15] ZHANG, B., WU, Y. L., LIU, Y. A. Wideband single-ended and differential bandpass filters based on terminated coupled line structures. IEEE Transactions on Microwave Theory and Techniques, 2017, vol. 65 no. 3 , p. 761-774. DOI: 10.1109/TMTT.2016.2628741

\section{About the Authors ...}

Yi-jun CAI was born in Fujian, China. He received the B.Sc. degree and Ph.D. degrees in Physical Electronics from Xiamen University, Xiamen, China, in 2010 and 2015, respectively. He is now a Lecturer with the Fujian Provincial Key Laboratory of Optoelectronic Technology and
Devices, School of Optoelectronic and Communication Engineering, Xiamen University of Technology, Xiamen, China. His research interests include microwave components and circuits, graphene-based terahertz devices, plasmonic devices.

Feng-yu ZHANG was born in Fujian, China. He received the B.Sc. degree in Fuzhou University, Fujian, China, in 2016, and currently he is working toward the M.S. degree in Xiamen University. His research interests include $\mathrm{RF} /$ microwave components and circuits.

Kai-da XU was born in Zhejiang, China. He received the B.S. and Ph.D. degrees in Electromagnetic Field and Microwave Technology from the University of Electronic Science and Technology of China (UESTC), Chengdu, China, in 2009 and 2015, respectively. From 2012 to 2014, he was a Visiting Researcher with the Department of Electrical and Computer Engineering, Duke University, Durham, NC, USA, under the financial support from the China Scholarship Council. From 2016 to 2017, he was a Postdoctoral Fellow with the State Key Laboratory of Millimeter Waves, City University of Hong Kong, Hong Kong. In 2015, he joined the Department of Electronic Science, Xiamen University, Xiamen, China as an Assistant Professor. Since 2018, he has been an Honorary Fellow with the Department of Electrical and Computer Engineering, University of Wisconsin-Madison, WI, USA. He has authored and coauthored over 90 papers in peer-reviewed journals and conference proceedings. He received the UESTC Outstanding Graduate Awards in 2009 and 2015, respectively. He was the recipient of the National Graduate Student Scholarship in 2012, 2013, and 2014 from the Ministry of Education, China. Since 2014, he has served as a reviewer for more than 30 journals including IEEE Transactions on Microwave Theory and Techniques, IEEE Transactions on Antennas and Propagation, IEEE Microwave and Wireless Components Letters and IEEE Antennas and Wireless Propagation Letters. Since 2017, he has served as an Associate Editor for both of the IEEE Access and Electronics Letters. He is also an Editorial Board Member of the AEÜ-International Journal of Electronics and Communications. His research interests include $\mathrm{RF} /$ microwave and $\mathrm{mm}$-wave circuits, antennas, and nanoscale memristors.

Dong-hao LI was born in Sichuan, China. He received his B.Sc. from Jilin University, Changchun, China, in 2016, and currently he is working toward the M.S. degree in Xiamen University. His research interests include $\mathrm{RF} /$ microwave components and circuits. 